\title{
Micro-scale Surface and Contaminate Modeling for Polarimetric Signature Prediction
}

\author{
M.G. Gartley, S.D. Brown and J.R. Schott \\ Digital Imaging and Remote Sensing Laboratory \\ Chester F. Carlson Center for Imaging Science \\ Rochester Institute of Technology \\ 54 Lomb Memorial Drive \\ Rochester, NY 14623-5604
}

\begin{abstract}
The optical properties of a surface may change significantly in response to contaminants from the environment and/or human activity. We utilize a first principles, physics-based radiometric ray tracing software package to evaluate the spectral polarimetric bi-directional reflectance distribution function (p-BRDF) of the virgin and contaminated surfaces. In the absence of contaminants and allowing only single bounces, we find the simulated reflectance properties of randomly rough Gaussian surfaces to be well represented by micro-facet based p-BRDF analytical models. However the addition of contaminants introduces phenomenology that falls outside the basic assumptions of the micro-facet analytical p-BRDF models. We will present initial results of p-BRDF simulations of random Gaussian surfaces with liquid contaminants.
\end{abstract}

Keywords: Polarization, remote sensing, image simulation, scene simulation, BRDF, contaminants

\section{INTRODUCTION}

Polarimatric imaging is a modality of current interest to the remote sensing community. Some have demonstrated the utility of polarimetric imaging in the areas of generic anomaly detection, land-mine detection, ${ }^{1}$ and oceanic wind speed determination. ${ }^{2}$ Generation of synthetic polarimetric scenes is useful for development of novel detection algorithms as well as design trades for future polarimetric collection systems. A critical component for modeling remotely sensed polarimetric scenes is the understanding of the polarized bi-directional reflectance distribution function (p-BRDF) of each scene element. The p-BRDF is commonly represented as a $4 \mathrm{x} 4$ element Mueller matrix transfer function. Each scene element's Mueller matrix describes how it radiometrically interacts with its surrounding environment, accounting for total radiance, linear polarization and circular polarization states.

Approaches to modeling the p-BRDF have evolved over the years, starting most notably with a classic paper ${ }^{3}$ by Torrance and Sparrow treating in-plane surface scattering as happening from a statistical slope distribution of mirror-like microfacets making up the surfaces' topography. Variants on this idea, such as the Beard-Maxwell and Germer-Priest microfacet models, have gained traction and demonstrated success for accurately capturing the phenomenology of measured p-BRDF data. In order to make the microfacet models more physically relevant, many authors have derived functional forms of shadowing and obscuration functions to attenuate the p-BRDF for increasingly rough surface textures. Although the micro-facet approach to p-BRDF modeling has roots in first principles physics, the utility of the models have been primarily to serve as empirical equations to capture phenomenology and not always physically relevant to actual surface texture and optic properties. This being said, predicting a p-BRDF for a new material based purely on knowledge of optical constants and surface texture is typically not possible due to many physical phenomena not captured in the micro-facet model assumptions.

\section{DERIVATION OF A GENERALIZED MICROFACET P-BRDF}

Fresnel reflection from a perfectly smooth mirror surface is well defined for both dielectric and metal surfaces. 


\subsection{Single Surface Flat to Ground}

Knowing the optical constants of the material allows one to leverage Fresnel coefficients to calculate in and out of plane polarized reflectivity, transmission, and emissivity. For dielectric materials characterized by a real valued index of refraction $n$, the Fresnel reflection coefficients are written as

$$
\begin{gathered}
r_{s}=\frac{n^{2} \cos \theta_{i}-\sqrt{n^{2}-\sin ^{2} \theta_{i}}}{n^{2} \cos \theta_{i}+\sqrt{n^{2}-\sin ^{2} \theta_{i}}} \\
r_{p}=\frac{\cos \theta_{i}-\sqrt{n^{2}-\sin ^{2} \theta_{i}}}{\cos \theta_{i}+\sqrt{n^{2}-\sin ^{2} \theta_{i}}}
\end{gathered}
$$

where the angle of incidence $\theta_{i}$ is the zenith angle relative to the surface normal direction. The Fresnel reflection coefficients for metal surfaces, characterized by a complex index of refraction, are slightly more complicated and written as

$$
\begin{gathered}
r_{s}=\frac{a^{2}+b^{2}-2 a \cos \theta_{i}+\cos ^{2} \theta_{i}}{a^{2}+b^{2}+2 a \cos \theta_{i}+\cos ^{2} \theta_{i}} \\
r_{p}=r_{s} \frac{a^{2}+b^{2}-2 a \sin \theta_{i} \tan \theta_{i}+\sin ^{2} \theta_{i} \tan ^{2} \theta_{i}}{a^{2}+b^{2}+2 a \sin \theta_{i} \tan \theta_{i}+\sin ^{2} \theta_{i} \tan ^{2} \theta_{i}}
\end{gathered}
$$

where the variables $a$ and $b$ are found in terms of the real and imaginary parts of the complex index of refraction, $\tilde{n}=n-i \kappa$ as follows

$$
\begin{aligned}
& 2 a^{2}=\sqrt{\left(n^{2}-\kappa^{2}-\sin ^{2} \theta_{i}\right)^{2}}+\left(n^{2}-\kappa^{2}-\sin ^{2} \theta_{i}\right) \\
& 2 b^{2}=\sqrt{\left(n^{2}-\kappa^{2}-\sin ^{2} \theta_{i}\right)^{2}}-\left(n^{2}-\kappa^{2}-\sin ^{2} \theta_{i}\right)
\end{aligned}
$$

Utilizing the Fresnel coefficients for a dielectric or metal, the reflectance factors determine the relationship between the incident and exitent irradiance is written as

$$
\begin{aligned}
& E_{r, s}=\left|r_{s}\right|^{2} E_{i} \\
& E_{r, p}=\left|r_{p}\right|^{2} E_{i}
\end{aligned}
$$

\subsection{Single Surface with Arbitrary Orientation}

The previously stated Fresnel coefficients are valid under the assumption that the surface of interest has a surface normal in the same direction as the global "up" direction of the incident irradiance and camera viewing coordinate system. Under the more general case of the material surface having an arbitrary orientation, the polarization state of the incident irradiance must be rotated into the local surface coordinate, Fresnel Reflection coefficients applied, and then rotated back into a global coordinate system. Under the Stoke's calculus formalism of representing polarization states of radiometric quantities, we write this relationship as

$$
S_{r}=R\left(-\alpha_{r}\right) F\left(\theta_{i}, n, \kappa\right) R\left(\alpha_{i}\right) S_{i}
$$

where the incident and reflected Stoke's vectors are $S_{i}$ and $S_{r}$ respectively and $R\left(-\alpha_{r}\right)$ and $R\left(\alpha_{i}\right)$ are the $4 \times 4$ Mueller rotation matrices to go from the global to surface local and global to surface local polarization coordinates respectively. The Fresnel reflection Mueller matrix $F\left(\theta_{i}, n, \kappa\right)$ and rotation matrix $R(\theta)$ can be written as

$$
F\left(\theta_{i}, n, \kappa\right)=\left(\begin{array}{cccc}
R_{p}+R_{s} & R_{p}-R_{s} & 0 & 0 \\
R_{p}-R_{s} & R_{p}+R_{s} & 0 & 0 \\
0 & 0 & 2 \operatorname{Re}\left(r_{p} r_{s}^{\star}\right) & 2 \operatorname{Im}\left(r_{p} r_{s}^{\star}\right) \\
0 & 0 & -2 \operatorname{Im}\left(r_{p} r_{s}^{\star}\right) & 2 \operatorname{Re}\left(r_{p} r_{s}^{\star}\right)
\end{array}\right)
$$




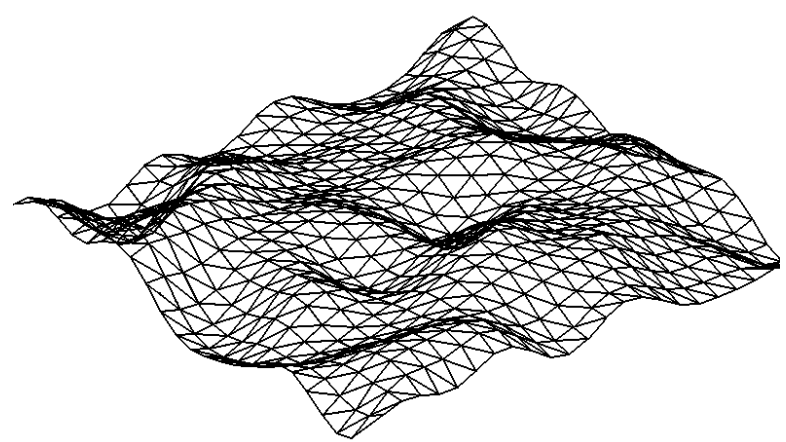

Figure 1. Triangulated micro-facet surface with correlation length of 0.06 , root mean square roughness of 0.02 , and surface slope variance of 0.47 radians.

$$
R(\theta)=\left(\begin{array}{cccc}
1 & 0 & 0 & 0 \\
0 & \cos 2 \theta & \sin 2 \theta & 0 \\
0 & -\sin 2 \theta & \cos 2 \theta & 0 \\
0 & 0 & 0 & 1
\end{array}\right)
$$

In order to determine the polarization coordinate system rotation angles $\alpha_{r}$ and $\alpha_{i}$ are determined with respect to the angle between the surface normal and the global normal $\theta_{N}$ and the scattering half angle $\beta$,

$$
\begin{gathered}
\cos \theta_{N}=\frac{\cos \theta_{i}+\cos \theta_{r}}{2 \cos \beta} \\
\cos \beta=\frac{\cos \theta_{i} \cos \theta_{r}+\sin \theta_{i} \sin \theta_{r} \cos \phi}{2}
\end{gathered}
$$

Finally, the rotation angles to orient the incident and exitent radiance from global to local coordinates are given by

$$
\begin{aligned}
\cos \alpha_{i} & =\frac{\cos \theta_{N}-\cos \theta_{i} \cos \beta}{\sin \theta_{i} \sin \beta} \\
\cos \alpha_{r} & =\frac{\cos \theta_{N}-\cos \theta_{r} \cos \beta}{\sin \theta_{r} \sin \beta}
\end{aligned}
$$

Therefore, the generalized Fresnel reflection coefficient Mueller matrix $F^{\prime}$ can be written as

$$
\left.F^{\prime}\left(\theta_{i}, \theta_{r}, \phi, n, \kappa\right)=\left[R\left(-\alpha_{r}\right) F\left(\theta_{i}, n, \kappa\right)\right)\right] R\left(\alpha_{i}\right)
$$

for any incident and reflected directions defined by the coordinates incident zenith $\theta_{i}$, reflected zenith $\theta_{r}$, and relative azimuth $\phi$ between the incident and reflected directions in the global coordinate system.

\subsection{Rough Multifaceted Surface}

Real world surfaces may be thought to be composed of a triangulated series of perfectly smooth micro-facets (Figure 1). Although the net effect is a rough surface, at a very small scale the surfaces are treated as Fresnel reflectors. A micro-facetized surface can be represented by a statistical distribution of angular slope orientations.

One such common statistical slope distribution has a Gaussian form

$$
P_{G}(\theta, \sigma, B)=\frac{B}{2 \pi \sigma^{2} \cos ^{3} \theta} e^{-\frac{\tan ^{2} \theta}{2 \sigma^{2}}}
$$

In many cases, the Gaussian form of the micro-facet slope distribution function is preferable for two reasons: (1) when coupled with a unity reflectance factor, it produces a unity directional hemispherical reflectance and (2) it is a good approximation to the surface texture of many common surfaces.

$$
\int_{0}^{2 \pi} \int_{0}^{\pi / 2} P_{G}(\theta, \sigma, 1) \sin \theta \cos \theta d \theta=1
$$


Another advantageous property of the Gaussian slope distribution function is its relationship to surface heights and correlation length. By definition, the surface height and correlation functions are both Gaussian in form and related to the rms surface slope by the relation

$$
\sigma=\frac{\sigma_{r m s} \sqrt{2}}{l_{c}}
$$

where $l_{c}$ is the correlation length and $\sigma_{r m s}$ is the root-mean-square of the surface heights. Other forms of the micro-facet slope distribution function are found in the literature, namely a modified Cauchy distribution function and a modified Gaussian distribution function. The reader is directed to the literature for more information on these specific functions.

Assuming the surface slope probability distribution function has a finite value for any desired facet orientation, the specular portion of a generalized micro-facet BRDF takes the form

$$
\rho_{s}=\frac{P(\theta, \sigma, b) F^{\prime}\left(\theta_{i}, \theta_{r}, \phi, n, \kappa\right)}{4 \cos \theta_{i} \cos \theta_{r}} S
$$

where $S$ is an arbitrary function which accounts for obscuration of the viewer and shadowing of the source by other facets. A simple version of a shadowing function takes the form of that presented by Maxwell and Beard,

$$
S\left(\theta_{N}, \beta, \tau, \Omega\right)=\frac{1+\frac{\theta_{N}}{\Omega} e^{-2 \beta / \tau}}{1+\frac{\theta_{N}}{\Omega}}
$$

This simplified form of the micro-facet BRDF assumes no secondary scattering effects such as volume and multiple facet scattering.

\subsection{Secondary Scattering Effects}

The simple, specular micro-facet BRDF presented in Equation 20 ignores radiance that may transmit through the micro-facet surface, scatter underneath the surface, and re-transmit through another facet towards the viewer. In addition, this simple BRDF ignores the radiance that is reflected from one micro-facet surface, towards another micro-facet surface, and then reflected towards the viewer.

An unpolarized volume scattering term has been introduced in the literature ${ }^{4}$ having the form

$$
\rho_{v}\left(\theta_{i}, \theta_{r}, n, \kappa, \alpha\right)=\alpha T\left(n, \kappa, \theta_{i}\right) T\left(n, \kappa, \theta_{r}\right)
$$

where $T\left(n, \kappa, \theta_{r}\right)$ corresponds to the unpolarized Fresnel transmission through an interface having a complex index of refraction $n-i \kappa$ and $\alpha$ is a weighting parameter representing the fraction of energy not absorbed by the material. For dielectric materials the $\alpha$ parameter approaches 1 , while for metals it approaches 0 .

An unpolarized multiple scattering term was also presented by the same authors, which contains a purely diffuse term and a geometrically dependent diffuse term

$$
\rho_{d}=\rho_{d 1}+\frac{\rho_{d 2}}{\cos \theta_{i} \cos \theta_{r}}
$$

where $\rho_{d 1}$ and $\rho_{d 2}$ are arbitrary constants. This form of a diffuse, multiple scatter term is purely empirical, but found to fit measured material properties quite well.

With the inclusion of the unpolarized multiple scatter and volume scattering terms, the full micro-facet BRDF takes on the form

$$
\rho=\rho_{s}+\rho_{v}+\rho_{d}
$$

and has units of steradians ${ }^{-1}$. 


\section{A RAY-TRACING APPROACH TO P-BRDF PREDICTION}

The previous section derived a functional form for a polarized BRDF based on physical principles. However, the final equation is a mix of terms based in physics (such as the Fresnel reflection matrix) mixed with empirical correction factors (such as the multiple scattering term and shadowing function). The utility of the generalized BRDF is that it permits a concise mathematical framework with which to polarimetrically model material reflections from a simple surface.

The main shortcoming of the generalized micro-facet BRDF is its inability to predict based purely on first principles optical properties and morphology. More specifically, the function is merely a statistical representation of a single material surface with surface slopes well described by a mathematical formula. This prompts multiple questions such as, how do we model a surface with a morphology not easily represented by a statistical slope distribution function? How do we model a material surface that contains contaminants of another material type or has spatially dependent optical properties? We could attempt to include more corrective terms and empirical scale factors into our generalized microfacet model, or we could step back and look at the problem as a computer graphics researcher might.

\subsection{DIRSIG}

The initial development of the Digital Imaging and Remote Sensing Image Generation (DIRSIG) model was begun at Rochester Institute of Technology (RIT) in the late 1980s as a 3D simulation environment for predicting images that would be produced by thermal infrared systems. Since that time, the model has been expanded to cover the 0.35 to 20.0 micron region of the spectrum. The model is designed to produce passive broad-band, multi-spectral and hyper-spectral imagery through the integration of a suite of first-principles based radiation propagation modules. These object oriented modules address tasks ranging from bidirectional reflectance distribution function (BRDF) predictions of a surface, to time and material dependent surface temperature predictions, to the dynamic viewing geometry of scanning imaging instruments on agile platforms. In addition to the myriad of DIRSIG specific objects that have been created, there is a suite of interface objects that leverage externally developed components (e.g. MODTRAN and FASCODE) that are modeling workhorses for the multi- and hyper-spectral community. The software is employed internally at RIT and externally within the user community as a tool to aid in the evaluation of sensor designs and to produce imagery for algorithm testing purposes. Key components of the model and some aspects of the models overall performance have been gauged by several validation efforts over the past decade of the models evolution..$^{5,6}$ The initial modification of the DIRSIG radiometry framework to support fully spectral-polarimetric radiation propagation was completed in 2002.

The DIRSIG model is a image generation tool that utilizes a complex computational radiometry sub-system to predict absolute fluxes within a 3D scene description. The model uses $\left[\begin{array}{lll}1 & \mathrm{x} & 4\end{array}\right]$ Stokes vector and $[4 \mathrm{x} 4]$ Mueller matrix calculus to propagate, reflect, transmit, etc. fluxes within the simulated scene environment. When modeling signatures the mid-wave infrared (MWIR) region (3-5 microns), the daytime illumination from the Sun is proportional to the emitted radiation from ambient (approximately $300 \mathrm{~K}$ ) materials. Furthermore, most materials have moderate reflectances (e.g. $\rho>0.4$ ) in the MWIR region and, therefore, we must consider both the reflected and the self-emission contributions to the surface leaving flux. The DIRSIG radiometry engine utilizes a single expression, governing equation across all wavelength regions such that reflected and self-emitted contributions are always included unless explicitly disabled.

The DIRSIG model has a flexible radiometry sub-system for computing radiances for arbitrary paths within the defined scene. The primary mechanism used to predict images is reverse ray-tracing where rays originate from the imaging detectors and are propagated into the scene. When a ray intersects the scene geometry, the associated radiometry solver is run to compute the surface leaving radiance. DIRSIG has a handful of radiometry solvers used for opaque surfaces and the most flexible is the "generic" radiometry solver. The generic radiometry solver computes the reflected radiance by sampling the hemisphere above the target. The distribution of these samples is based on the shape and magnitude of the associated BRDF. The nominal hemispherical sampling is cosine projected and has user-defined sampling parameters (e.g. total number of samples, etc.). The incident

load for those samples are determined by tracing higher generation rays which intersect other surfaces and trigger other instances of the radiometry solver. The fidelity of the sampling for higher generation bounces can be decreased using a bounce-dependent decay rate that modifies the sampling parameters associated with that 


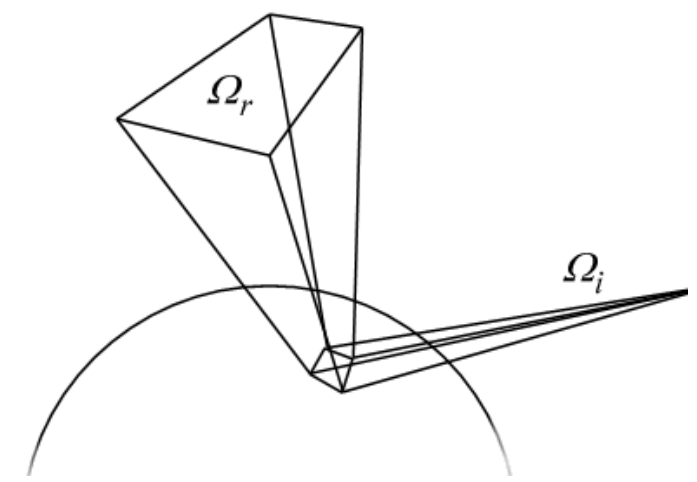

Figure 2. Schematic demonstrating the change in detector solid angle upon reflection from a curved surface.

surface. The total number of bounces that are tracked is also user controllable. The incident loads from the sampled hemisphere are numerically integrated using the geometry specific reflectance (BRDF) and the solid angle of the sample. The incident illumination from the Sun, Moon and sky are provided by MODTRAN-P which has been integration tested with DIRSIG against Coulsons polarized sky measurements. ${ }^{7}$ Since the p$\mathrm{BRDF}$ is accessed via surface relative incident and reflected angles, the polarization orientation of the incident illuminates must be projected into the surface oordinate system, and the leaving radiance must be projected into the polarization coordinates of the scene. ${ }^{8}$

\subsection{Propagation of Ray Solid Angle}

Reflection from a scene facet attributed with an analytical p-BRDF requires sampling of the irradiance incident on the facet from the entire hemisphere above it, weight by the p-BRDF. However, treating a facet as perfectly specular (Fresnel reflection) results in a delta function p-BRDF. Under this condition, the solid angle (in units of steradians) may also change upon reflection.

By default, DIRSIG facets are treated as locally flat. This permits the propagation of a constant detector solid angle between specular facets.However, many surfaces are not locally flat across the span of a single detector element instantaneous field of view (IFOV), especially those containing spherical contaminant particles. Therefore, a modified version of the DIRSIG model can project a ray from the detector center as well as the 4 corners of a detector element. The reflected detector corner rays determine the solid angle utilized to sample the hemisphere above a facet, as well as the gain/attenuation applied to incident irradiance. This feature of the modified DIRSIG model is key to capturing specular source glints (a key capability for p-BRDF measurement simulation) as well as maintaining the radiometric integrity of the simulation.

\section{MODEL VERIFICATION}

In this section we describe a detailed effort to verify the micro-facet p-BRDF simulation of simple surfaces against the analytical form of the p-BRDF presented in Section 2. In addition, we also present experimental results for p-BRDF measurements conducted on vinyl siding and compare them to predictions from the analytical p-BRDF model.

\subsection{Comparison to Analytical Model}

Gaussian surface height maps were created utilizing a technique derived from the approach described by Fenton. ${ }^{9}$ This technique is essentially applying a white noise gain to the desired surface auto-correlation function, with careful attention paid to the symmetry of the noise pattern.

As mentioned earlier, the authors prefer utilizing the Gaussian surface slope distribution assumption due to its convenient mathematical properties. An example of a Gaussian height field image is presented in Figure 3 which has been contaminated with spherical particles. The addition of the particles is accomplished through a secondary tool developed in IDL. 


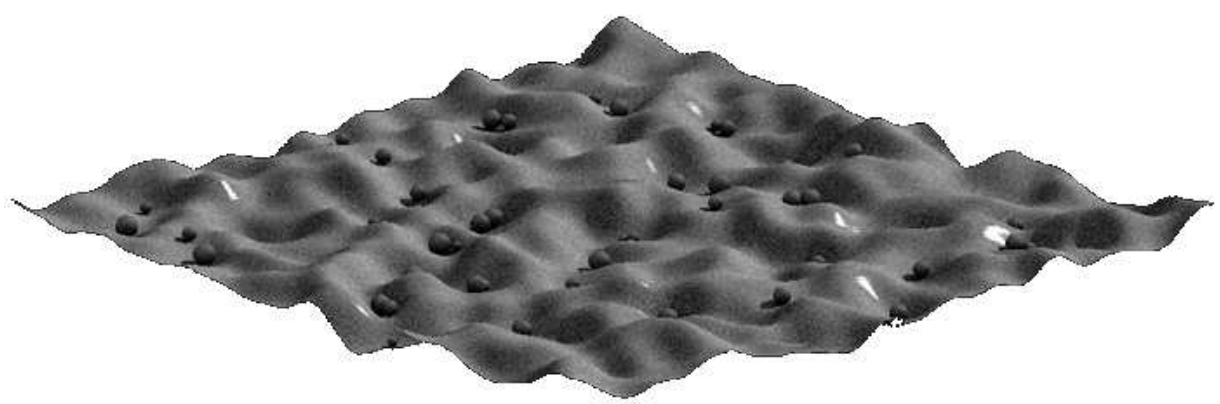

Figure 3. Example of a randomly rough Gaussian height field surface with a root mean square roughness of 2 relative units, surface correlation length of 15 relative units, and rms slope of 0.05 radians.
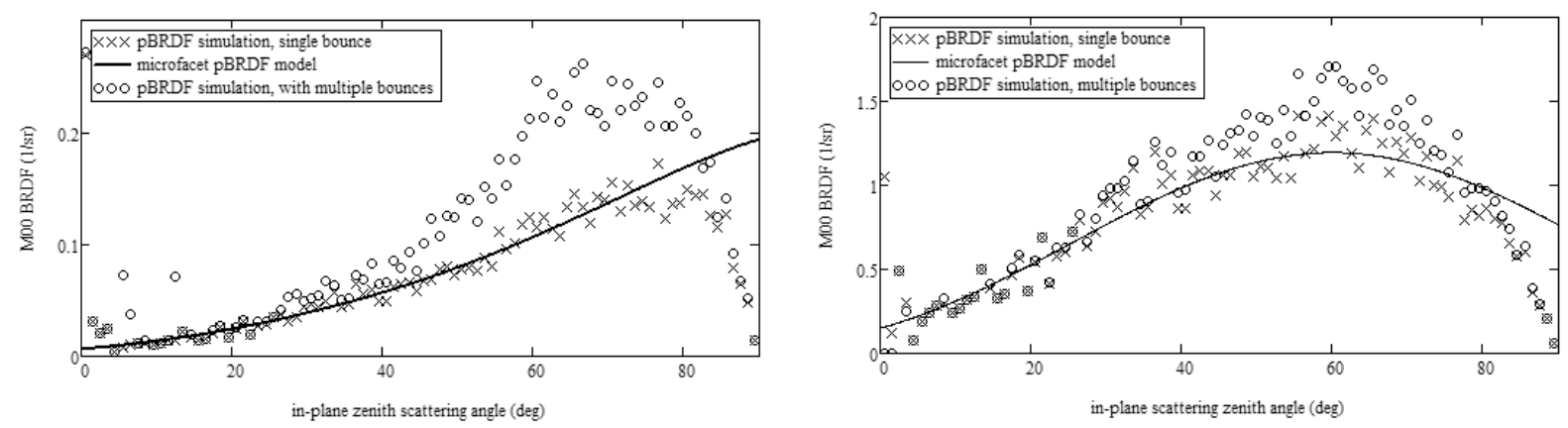

Figure 4. Triangulated micro-facet surface with correlation length of 0.06 , root mean square roughness of 0.02 , and surface slope variance of 0.47 radians.

For the purposes of model verification, we examine a clean micro-facetized surface with a Gaussian height distribution. We expect the single bounce results to match the analytical form of the appropriately configured p-BRDF described in Section 2. The micro-surface p-BRDF simulation was conducted on randomly rough Gaussian surfaces with rms surface slope values of $0.05,0.15$, and 0.30 radians. The scattering Mueller matrices were generated for incident zenith angles of 10.0, 41.4, and 60.0 degrees on surfaces assigned real valued index of refraction values of $n=1.5$ (representative of glass and many plastic materials) and $n=1 \cdot 10^{6}$ (representative of a perfectly reflecting dielectric).

We found good agreement between the micro-surface simulated p-BRDF Mueller scattering matrices and the analytical form of the p-BRDF within the statistical noise of the limited number of micro-facets. However, the rougher surfaces showed shadowing effects in the simulated p-BRDF magnitudes at oblique source and view geometries not captured in the analytical form of the p-BRDF model. Although the analytical form of the p-BRDF model is capable of being configured with a shadowing and source obscuration function, we have not yet integrated the statistically based shadowing functions found in the literature into the model at this time. The shadowing functions that are currently available to us are purely empirical and utilized to fit experimentally measured data, not to predict shadowing effects of notional surfaces as we are currently investigating.

The p-BRDF simulation model is capable of distinguishing between single and multiple bounce scattering effects permitting an apples to apples comparison against the analytical p-BRDF model. The two plots in Figure 4 show the single and multiple bounce scattering magnitudes for both the $n=1.5$ and $n=1 \cdot 10^{6}$ surfaces having rms slope values of 0.30 radians at an incident zenith angle of 60 degrees. There is a significant boost in the total scattering magnitude due to multiple bounce effects for the $n=1.5$ surface and a moderate boost in scattering magnitude for the $n=1 \cdot 10^{6}$ surface. The dependence of the multiple bounce scattering boost on micro-facet index of refraction is understood by examination of the multiple bounce paths. These paths typically have a secondary bounce that arrives at a close to grazing angle which has a much higher reflectivity value compared to the initial bounce for low index of refraction micro-facets. However, for micro-facets with a large index of refraction, the reflectivity is almost identical across the range of incidence angles ranging from nadir to grazing resulting in a smaller overall scattering boost. 


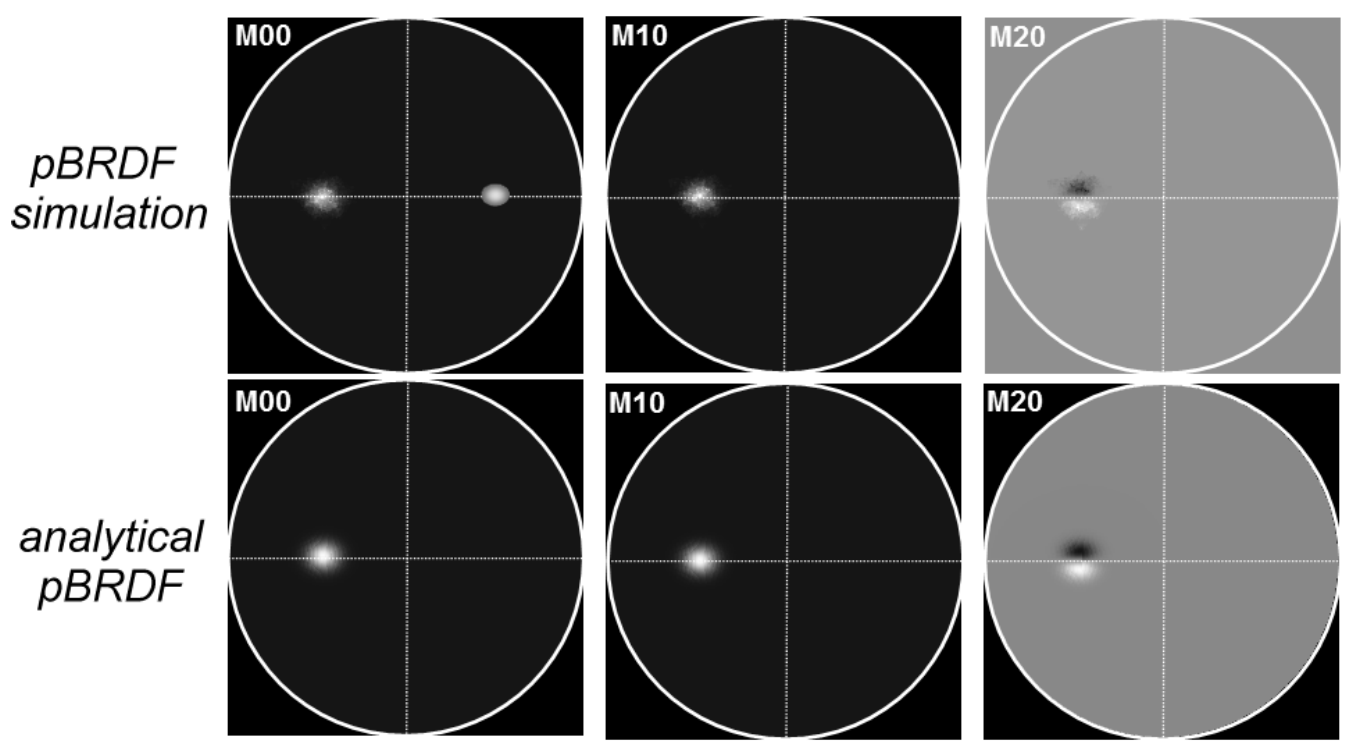

Figure 5. Polar plots of the Mueller scattering matrix elements M00 (left), M10 (center), and M20 (right) derived from the micro-facetized surface p-BRDF simulation (top row) and the analytical form (bottom row) of the p-BRDF for a clean, randomly rough Gaussian surface with $\mathrm{rms}$ slope value of $0.05 \mathrm{rad}, n=1.5$, and angle of incidence of 41.4 degrees.

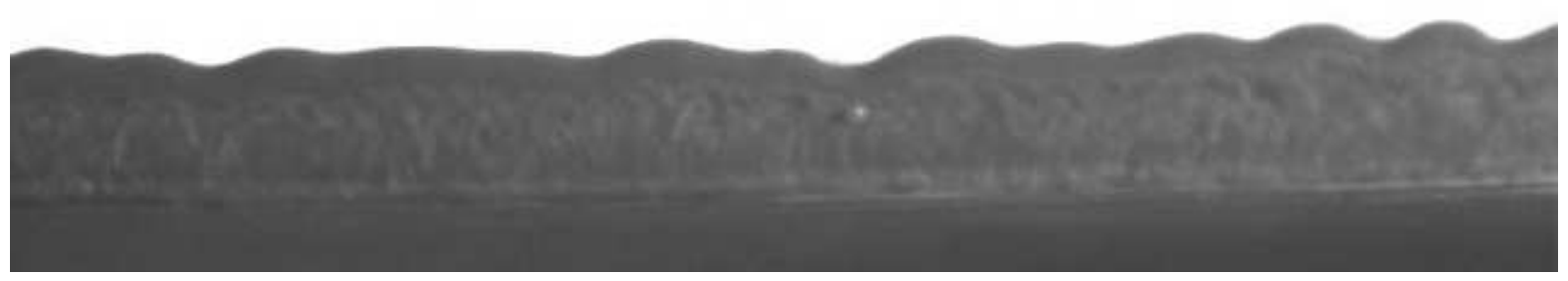

Figure 6. An optical microscope image of the side profile of vinyl siding.

We present another example of the verification effort for the case of a randomly rough Gaussian surface with a rms slope value of 0.05 radians, index of refraction $n=1.5$, and angle of incidence of 41.4 degrees in Figure 5 .

\subsection{Comparison to Measured Data}

Now that we have verified the radiometric integrity of the micro-facet based p-BRDF simulation against an analytical model, we next compare the p-BRDF predictions for a simple surface against experimentally measured data. It is common in the literature to verify models against surfaces such as bead-blasted aluminum or glass. However, we have chosen the surface of vinyl siding as material for investigation because it is much more likely to have relevance to the remote sensing community.

Optical microscopy was utilized to acquire surface height profiles of a sample of vinyl siding (see Figure 6). The measured height data was found to fit a Gaussian height distribution with rms roughness of 2.6 (relative units), auto-correlation length of 14.5 (relative units) and rms slope of 0.252 radians. Utilizing the measured rms surface slope of 0.252 radians, we configured the analytical p-BRDF model with index of refraction representative of PVC $(n=1.53)$.

The plots in Figure 8 demonstrate the agreement in the shape of the M00, M10 and DOLP curves in the plane of incidence for the source at a 30 degree zenith angle. We were not able to independently decouple the magnitude of the specular and diffuse scattering terms in the measured p-BRDF. Therefore, we "assumed" a $13 \%$ diffuse Lambertian reflectivity and gain of 0.0011 on the polarizing specular reflectance term. Therefore, the magnitude of the degree of linear polarization curve has been fit by hand, but the shape of the curve is driven solely by the measured rms slope value of 0.252 radians (see right hand side of Figure 8 ). This verification of the p-BRDF model (both analytical and Monte Carlo micro-facet based predictions) against experimental 

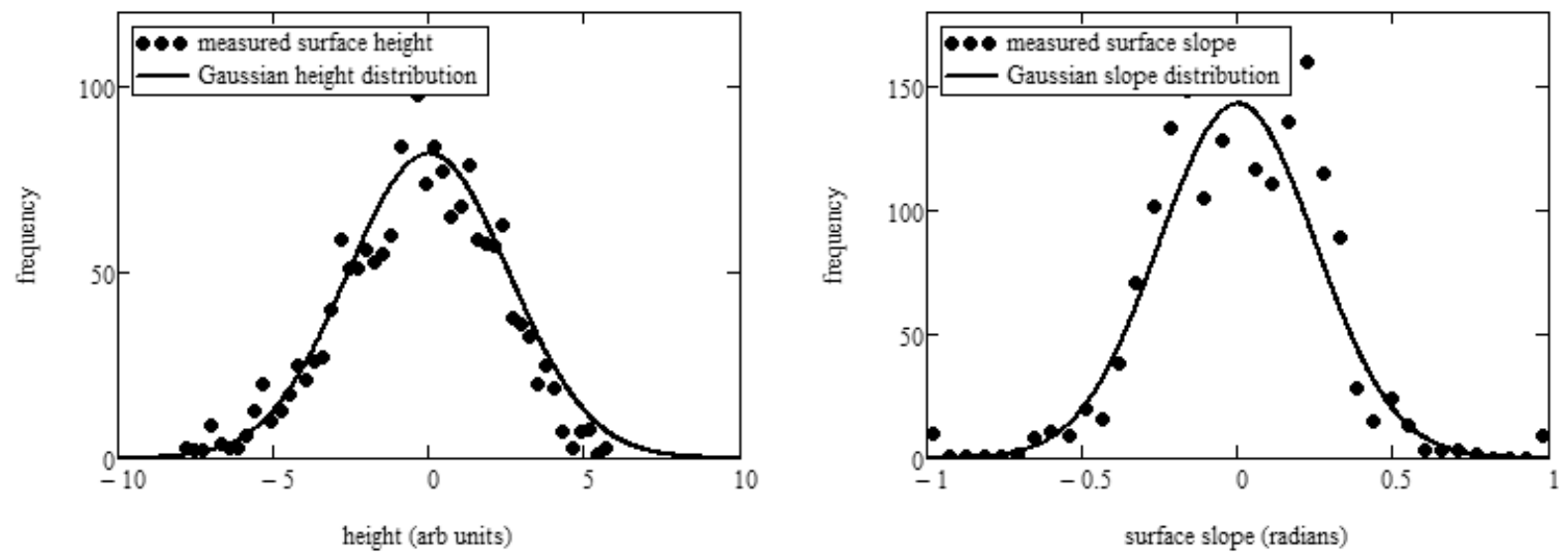

Figure 7. Surface height (left) and slope (right) histograms for vinyl siding in the rough direction. The measured data was found to fit a Gaussian height distribution with rms roughness of 2.6, correlation length of 14.5 and rms slope of 0.252 radians.
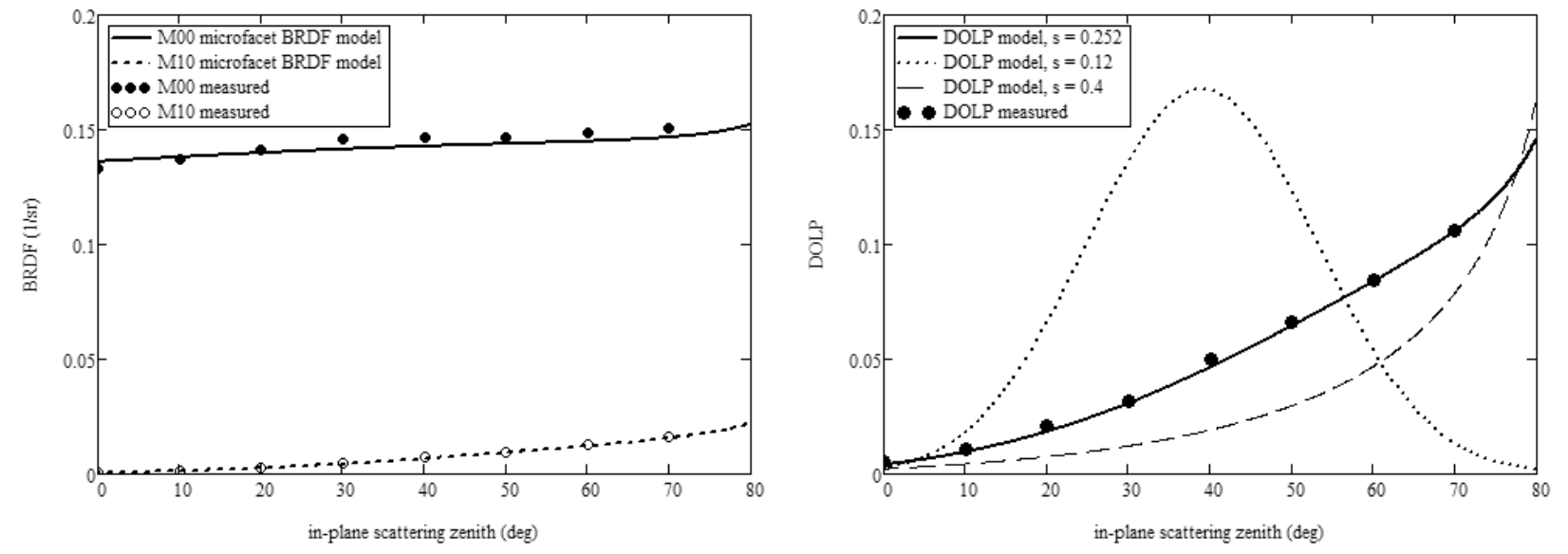

Figure 8. Measured and predicted M00, M10 (left) and DOLP (right) for vinyl siding in the plane of incidence in the rough surface direction. 

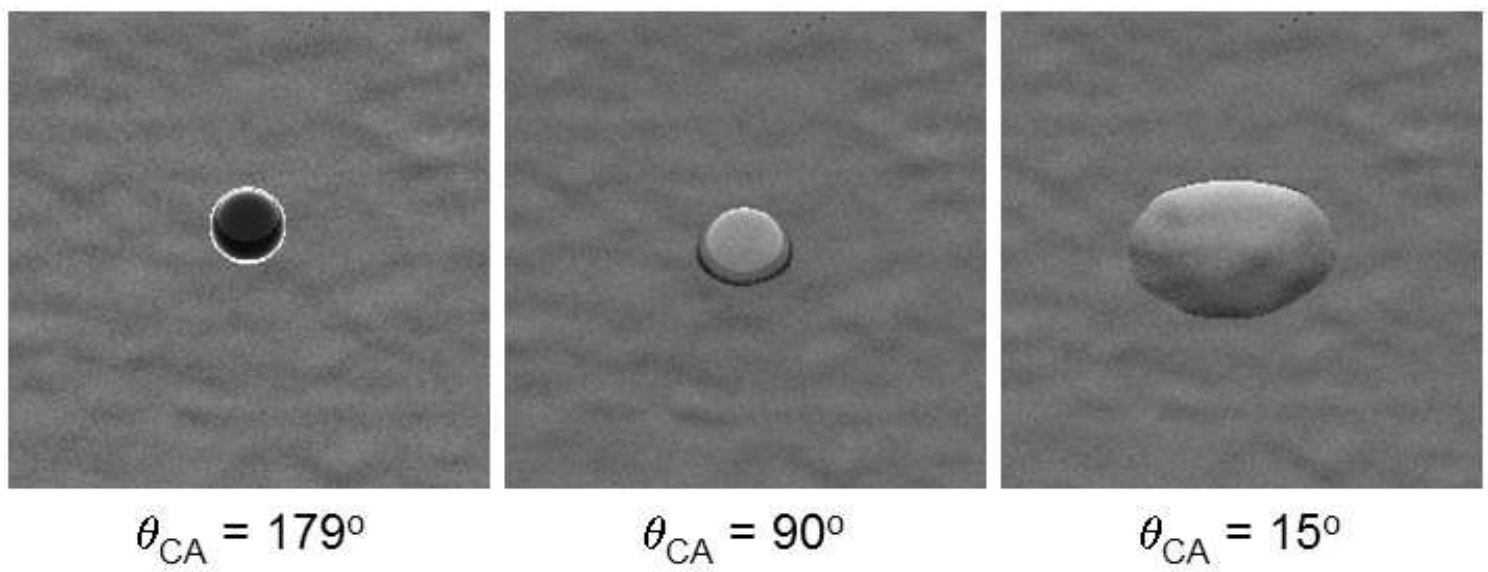

Figure 9. Visualization of a randomly rough Gaussian surface in the presence of water contamination (dew). Three realizations of the surface are visualized, each with a unique surface tension and resulting water contact angle.

measurements is meant to demonstrate the physical relevance of modeling actual surface morphology to make predictions of polarimetric phenomenology as a function of view geometry.

\section{INITIAL RESULTS FOR COMPLEX SURFACES}

The previous section verified the validity of utilizing either an analytical p-BRDF or a Monte Carlo ray-tracing approach to p-BRDF predictions for clean surfaces. Next, we examine a complex surface for which the analytical p-BRDF approach is not applicable and the utility of the Monte Carlo p-BRDF prediction is demonstrated.

Consider the case of a randomly rough Gaussian surface that is relatively smooth ( $\mathrm{rms}$ slope $=0.05 \mathrm{rad})$. We have applied a fixed volume of water $(n=1.333)$ to the surface and varied the surface tension of the micro-facet surface such that the water made a contact angle of 179, 90, and 15 degrees. A contact angle of 179 degrees indicates a very hydrophobic surface while a contact angle of 15 degrees indicates a very hydrophyllic surface. The question we wish to address in this example is how would an engineer tailor the surface tension of a painted surface with the intention of minimizing the enhancement of its polarimetric signature in the presence of morning dew. To answer the question, we ran Monte Carlo simulations with our micro-facet based p-BRDF simulation model to predict the M00, M10, and M20 elements of the Mueller scattering matrix. These results are presented in Figure 10.

The p-BRDF simulation results demonstrate that relatively hydrophobic surfaces show polarimetric signatures (captured in the M10 and M20 elements of the Mueller scattering matrix) due to the addition of dew dispersed somewhat uniformly and weakly across the entire hemisphere above the surface. However, the hydrophyllic surface shows a significant enhancement in the polarimetric signature around the specular lobe of the surface substrate effectively "polarimetrically lighting up" the surface in the presence of dew. Therefore, our p-BRDF prediction model analysis of this notional engineering problem has shown that hydrophobic surfaces are desirable for reducing polarimetric signature enhancement in the presence of dew.

\section{SUMMARY}

We have presented the framework for a new p-BRDF prediction tool leveraging the radiometric ray-tracing framework of DIRSIG. The predictions from the tool have been verified for clean, randomly rough surfaces against a generalized, analytical p-BRDF model. The physical relevance of our approach to predicting polarimetric signatures is demonstrated by agreement between predictions and experimental measurements of vinyl siding. Finally, we utilize the rigorous ray-tracing strengths of our model to demonstrate its utility to aid a materials engineer in the design of a painted surface to minimize polarimetric signature enhancement in the presence of dew. 

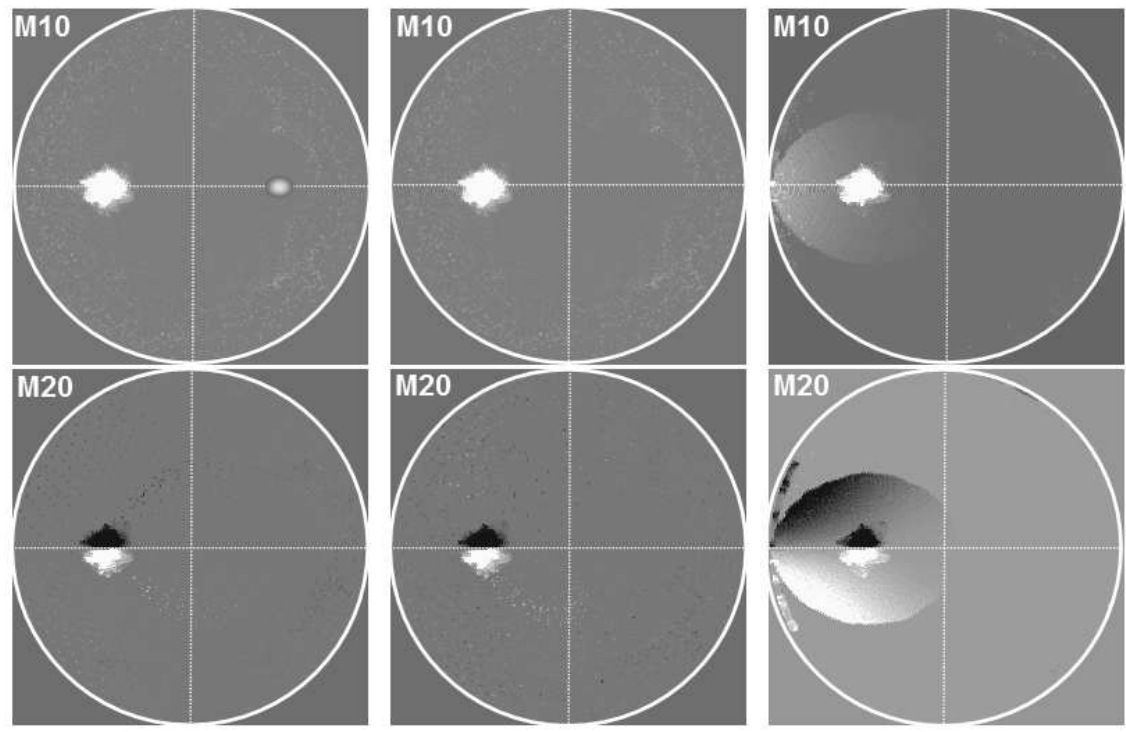

$\theta_{\mathrm{CA}}=179^{\circ}$

$\theta_{\mathrm{CA}}=90^{\circ}$

$\theta_{\mathrm{CA}}=15^{\circ}$

Figure 10. Polar plots of the Mueller scattering matrix elements M10 (top), M20 (bottom) for dew contaminated surfaces engineered to have a water contact angle of 179 (left), 90 (center), and 15 degrees (right).

\section{REFERENCES}

[1] Cremer, F., Jong, W., and Schutte, K., "Infrared polarization measurements and modeling applied to surfacelai antipersonnel landmines," Optical Engineering 41(5), 1021-1032 (2002).

[2] Monaldo, F., "Comparison of sar-derived wind speed with model predictions and ocean buoy measurements," IEEE Trans. Geoscience and Remote Sensing 39(12) (2001).

[3] Torrance, K. and Sparrow, E., "Theory for off-specular reflection from roughned surfaces," Journal of Optical Society of America 57(9) (1967).

[4] [Nonconventional Exploitation Factors Data System (NEFDS) Modeling Document v10], National Geospatial Intelligence Agency (2006).

[5] Brown, S. D., Schott, J. R., Raqueno, R. V., Kraska, T., and White, R., "Validation and analysis of the digital imaging and remote sensing laboratorys synthetic image generation models," Proc. of the Seventh Annual Ground Target Modeling and Validation Conference (1996).

[6] Mason, J. E., Schott, J. R., and Rankin-Parobek, D., "Validation analysis of the thermal and radiometric integrity of rits synthetic image generation model, dirsig," Proc. of SPIE, Characterization and Propagation of Sources and Backgrounds 2223 (1994).

[7] Devaraj, C., Brown, S., Messinger, D., Goodenough, A., and Pogorzala, D., "A framework for polarized radiance signature prediction for natural scenes," Proc. of SPIE, Algorithms and Technologies for Multispectral, Hyperspectral, and Ultraspectral Imagery XIII 6565 (2007).

[8] Gartley, M., Brown, S., and Schott, J., "Polarimetric scene modeling in the thermal infrared," Proc. of SPIE, Polarization Science and Remote Sensing III 6682 (2007).

[9] Fenton, G., "Error evaluation of three random field generators," Journal of Engineering Mechanics 120(12), 2478-2497 (1994). 